\title{
Improving the Efficiency of Opportunistic Routing in Dynamic Ad Hoc Networks by Eliminating Duplicate Message Forwarding
}

\author{
Varun G Menon \\ SCMS School of Engineering and Technology, Kerala, India 683582, \\ Email: varunmenon@scmsgroup.org
}

\begin{abstract}
Opportunistic routing has emerged as one of the prominent solutions for efficient data delivery in ad hoc networks especially in highly dynamic scenarios. Opportunistic routing uses the broadcasting nature of the wireless medium to increase the number of potential forwarding nodes in the network, thereby improving the delivery rate. But most of the proposed opportunistic routing protocols suffer from increased duplicate data forwarding at the intermediate nodes in the network which leads to increase in traffic and reduced efficiency. We propose a novel method which uses acknowledgements for controlling the data forwarding by intermediate nodes and thereby eliminating most of the duplicate forwarding in the network. Simulation results show that our proposed method is much more efficient that all the major existing opportunistic protocols.
\end{abstract}

\section{Keywords}

Duplicate Forwarding; Dynamic Ad Hoc Networks; Opportunistic Routing; Performance Improvement

\section{Introduction}

Mobile ad hoc networks (MANETs) [1-10] are a group of self-organized devices that can communicate with each other without the help of any centralized control or infrastructure. MANETs have brought a revolution in designing communication based applications especially for disaster recovery scenarios. Efficient routing and reliable delivery of packets from source to the destination have remained a major challenge. The recently proposed opportunistic routing protocols [11-12] have found to give better results compared to the earlier topology based protocols [13-16] and geographic routing protocols [17-21]. Recently many studies have been 
carried out on the performance of opportunistic routing protocols [22-35] in dynamic ad hoc networks. Many issues and challenges have been put forward in recent research in opportunistic protocols. This paper proposes a novel method to overcome one such major issue with opportunistic routing.

One of the major issues contributing to the transmission inefficiency and low Quality of Service (QoS) of opportunistic routing protocols in dynamic ad hoc networks is the duplicate or redundant forwarding of data packets at the intermediate devices. Duplicate forwarding of the data packet by devices other than the best forwarder leads to increase in traffic and congestion in the network. Also it is very important to restrict unwanted forwarding of data packet to minimize the energy usage of devices in the network.

This research article proposes an enhanced opportunistic routing technique, Control Relaying with Acknowledgements (CRA) that helps to eliminate the issue of duplicate data forwarding at intermediate nodes by controlling the relaying of data packets at each intermediate device in the network. This is done per data packet basis using information piggybacked on each packet. Only the devices that reply with an acknowledgement are considered for forwarding the data packet. Among the devices that reply with the acknowledgement, the device that is nearest to the destination is selected for forwarding. All the remaining devices do not act on the broadcasted data packet. Control Relaying with Acknowledgements (CRA) method enhances the efficiency of opportunistic routing by removing redundant data forwarding at intermediate devices and helps to achieve excellent Quality of Service dynamic ad hoc networks.

\section{Proposed Method: Control Relaying with Acknowledgements (CRA)}

Figure 1 illustrates the working of the proposed method Control Relaying with Acknowledgements (CRA). A highly dynamic ad hoc network is considered with 5 wireless devices S, A, B, C and D. All the devices in the network are highly mobile and they move randomly in the network. Here source device $S$ wants to send data packets to the destination device D. Assumption is made that each device is aware of its position and the position of its direct neighbours. Using the opportunistic routing strategy, a priority list of forwarders based on their nearness to the destination device is generated. So the list would be $\mathrm{C}, \mathrm{B}$, $\mathrm{A}$ in the descending order of their priority. The proposed method CRA, then piggyback a new field in the data packet header which is shown in table 1 . The initial sequence values (sequence numbers) for 
devices $\mathrm{A}, \mathrm{B}, \mathrm{C}$ would be 0 in data packet P1. Using the broadcasting property of the wireless network, device S broadcasts the data packet P1 intended for the destination device D.

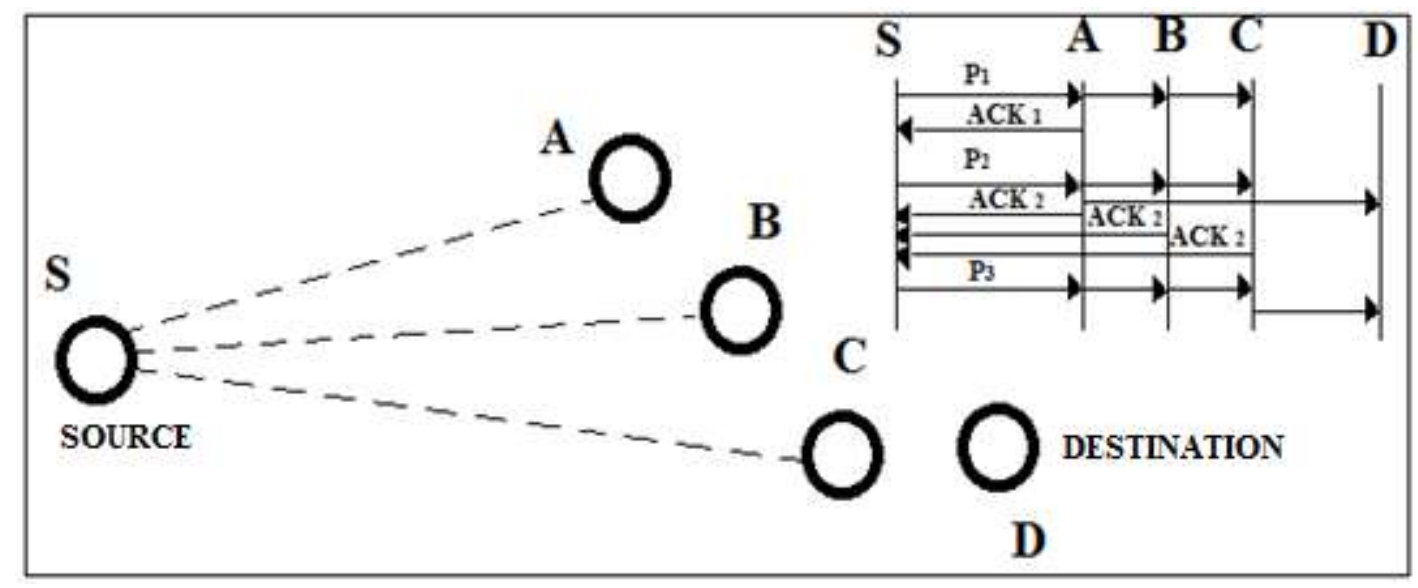

Figure 1 Working of CRA

Here the source device S receives acknowledgement for data packet P1 from device A only. This may be caused due to mobility of devices B and C. Also device A does not forward the data packet immediately and it waits for subsequent packets. Now the source device modifies the sequence value of device $\mathrm{A}$ to 1 in the special field and piggybacks it to packet P2 and broadcasts the data packet. The data packet $\mathrm{P} 2$ is received by all the three devices. Device A forwards the data packet P1 as soon as it receives the data packet P2 with sequence value 1 ( 1 is the sequence number of data packet P1). The major advantage is that devices $\mathrm{A}$ and $\mathrm{C}$ do not forward the data packet because the sequence value for those devices in data packet P2 is 0 and thus duplicate forwarding of data packets by these devices is eliminated. Now source device $\mathrm{S}$ receives acknowledgements from all the three devices for packet P2. Source device modifies the sequence value table and gives value 2 for device $\mathrm{C}$ which is the device that is nearest to the destination among the three. This value is piggybacked in packet P3. Device C forwards the data packet P2 as soon as it receives packet P3 with value 2. Using this technique only the best forwarder device forwards the data packet and thus we eliminate duplicate forwarding of data packets at the other devices in the network. 
Table 1 CRA Sequence values

\begin{tabular}{|c|c|c|c|c|c|}
\hline \multicolumn{2}{|c|}{ Packet P1 (Initial Value) } & \multicolumn{2}{|c|}{ Packet P2 } & \multicolumn{2}{c|}{ Packet P3 } \\
\hline Device & $\begin{array}{c}\text { Sequence } \\
\text { Value }\end{array}$ & Device & $\begin{array}{c}\text { Sequence } \\
\text { Value }\end{array}$ & Device & $\begin{array}{c}\text { Sequence } \\
\text { Value }\end{array}$ \\
\hline A & 0 & $\mathrm{~A}$ & 1 & $\mathrm{~A}$ & 1 \\
\hline $\mathrm{B}$ & 0 & $\mathrm{~B}$ & 0 & $\mathrm{~B}$ & 0 \\
\hline $\mathrm{C}$ & 0 & $\mathrm{C}$ & 0 & $\mathrm{C}$ & 2 \\
\hline
\end{tabular}

\section{RESULTS AND DISCUSSION}

Performance analysis of Control Relaying with Acknowledgements (CRA) method is done using simulations in NS-2. The performance of this method is compared with the latest and most popular opportunistic routing protocols in dynamic ad hoc networks.

\subsection{Simulation Parameters}

Simulation parameters used in the performance analysis of Control Relaying with Acknowledgements method is similar to those used with Reliable Routing Technique (RRT). IEEE $802.11 \mathrm{~g}$ is set as the MAC protocol for the simulation. The simulation starts with the deployment of 100 nodes in a network area of $1000 \times 800 \mathrm{~m}^{2}$ rectangular region. $250 \mathrm{~m}$ is set as the transmission range of various nodes in the network. Constant Bit Rate (CBR) traffic is being generated between the source to the destination nodes in the network at a rate of 20 packets per second $(40 \mathrm{kbps})$. The size of the data packet is set at 512 bytes. The simulation starts at 100 seconds and ends at 900 seconds. Mobility in the network is created by varying the speed of nodes from $5 \mathrm{~m} / \mathrm{s}$ to $50 \mathrm{~m} / \mathrm{s}$.

\subsection{Performance Metrics}

- Data Forwarding Times at Each Hop

Packet Forwarding Times per Hop measures the number of times a data packet is forwarded for delivery over each hop in the network. This metric gives a clear picture on the amount of redundant data transmissions happening at the intermediate devices in the network. Packet 
Forwarding Times per Hop is a very important performance metric measuring the quality and efficiency of a routing protocols in Dynamic ad hoc networks with extremely dynamic devices.

\subsection{Mobility Models}

To generate highly dynamic ad hoc networks in simulations it is very essential to use the appropriate mobility models. Different mobility models are used to generate different patterns of random movement and mobility of devices in Dynamic ad hoc networks. Generating different scenarios with different mobility models helps to test the performance of the proposed methods in multiple dynamic conditions. In order to assess the performance of the proposed methods in all types of mobile and dynamic environments three different mobility models are used.

- Random Way Point Mobility Model

- Random Walk Mobility Model

- Random Direction Mobility Model

\subsection{Protocols Selected for Comparison}

- $\quad$ ExOR

Extremely Opportunistic Routing (ExOR) is the most referenced and popular OR protocol. It was the first protocol to utilize the OR concept and offered better reliability compared to previous protocols. Many opportunistic routing protocols were proposed based on the working of this protocol.

\section{- CAOR}

Context Aware Opportunistic Routing (CAOR) is selected for comparison because it is the latest opportunistic protocol that gives maximum data delivery in the network. This protocol has very good performance compared to all the previous existing opportunistic routing protocols in HDMANETs with extremely dynamic wireless devices.

\section{- LCOR}

Least Cost Opportunistic Routing (LCOR) protocol generated the most optimized candidate set among all the OR protocols in HDMANETs. LCOR gave good performance 
compared to all optimization based opportunistic routing protocols in ad hoc network with extremely mobile devices.

\subsection{Simulation Results}

Each result discussed here is an average value calculated from 10 simulation runs in Network Simulator-2

\subsubsection{Variation of forwarding times per hop with speed}

Once the dynamic ad hoc network scenario is developed and the simulation parameters are set, Constant Bit Rate (CBR) traffic is generated between the source and destination devices at a rate of 20 packets per second. Initially the speeds of the devices are varied in the network from $5 \mathrm{~m} / \mathrm{s}$ to $40 \mathrm{~m} / \mathrm{s}$ and the corresponding Packet Forwarding Times per Hop value is measured for each opportunistic protocol separately in the network.

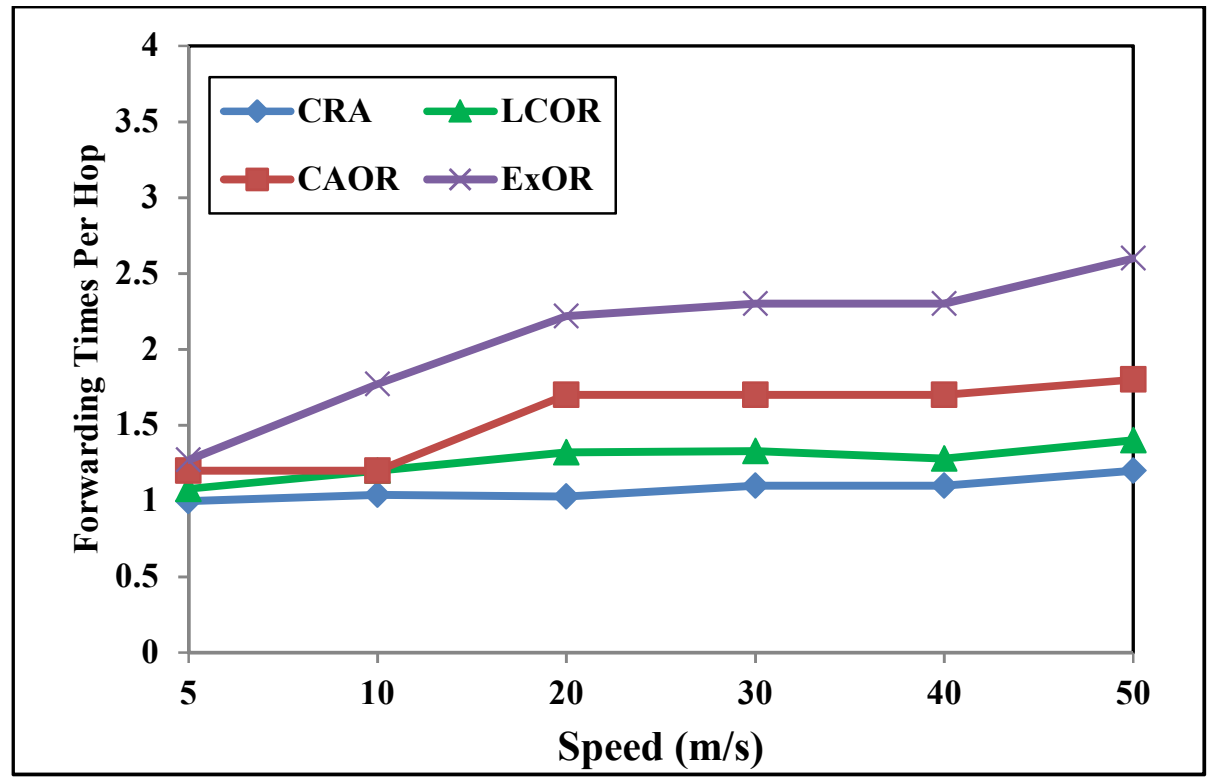

Figure 2 Forwarding Times Per Hop with Varying Speed of Nodes (Random Way Point Mobility Model) 


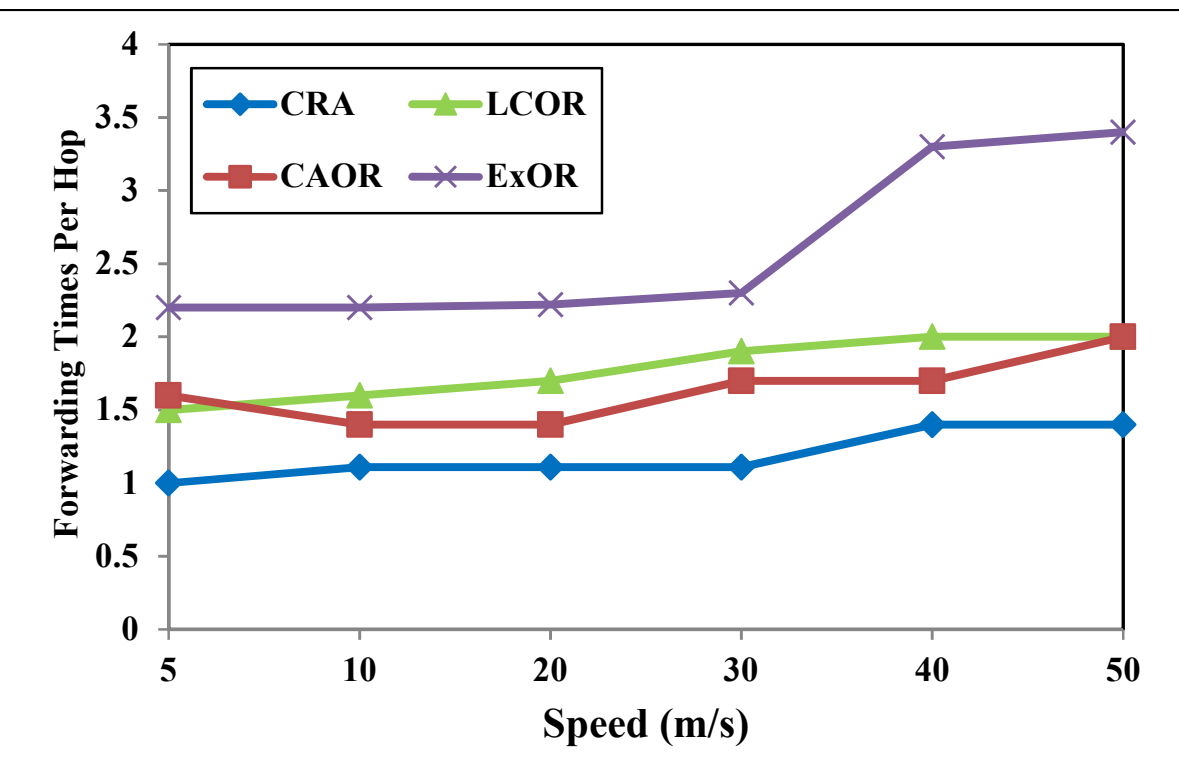

Figure 3 Forwarding Times Per Hop with Varying Speed of Nodes (Random Walk Mobility Model)

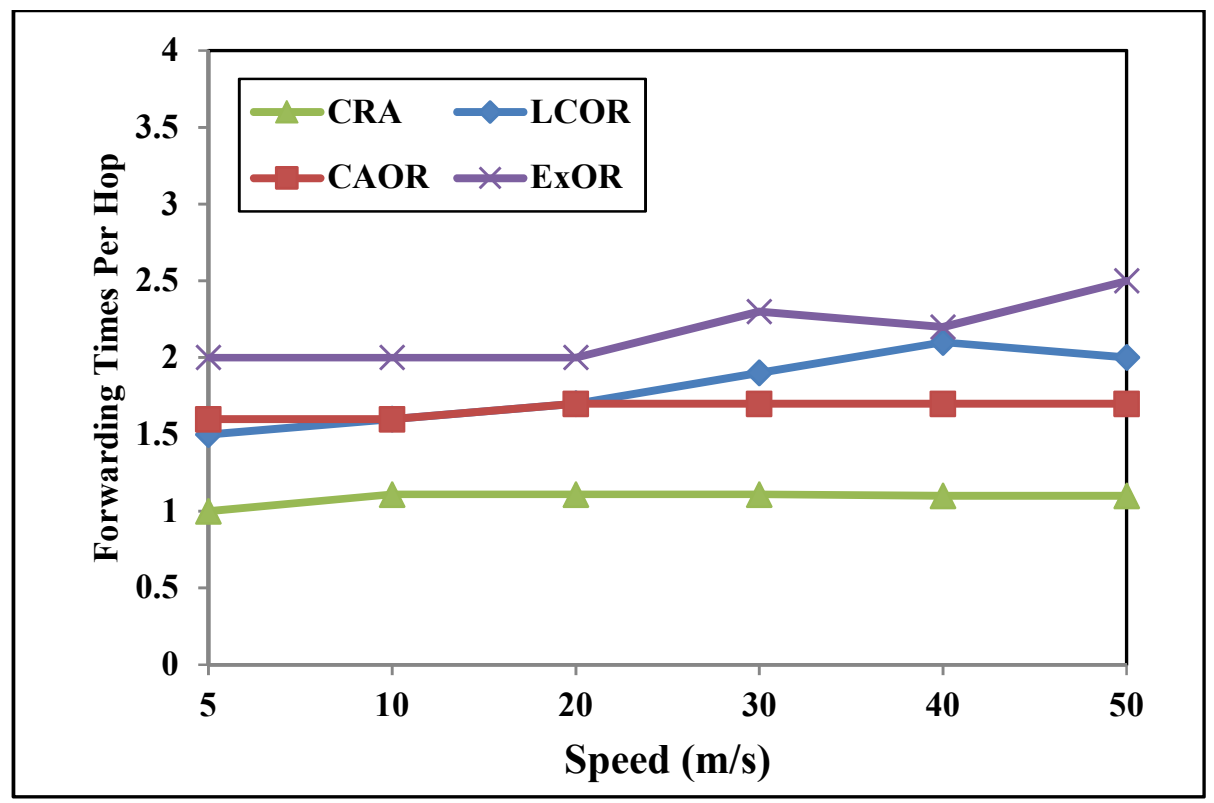

Figure 4 Forwarding Times Per Hop with Varying Speed of Nodes (Random Direction Mobility Model)

Figure 2, 3 and 4 shows the Packet Forwarding Times per hop value with varying speed of nodes obtained using Control Relaying with Acknowledgements, CAOR, LCOR and 
ExOR protocols in three simulations scenarios generated by random way point mobility model, random walk mobility model and random direction mobility model respectively. From the graph it is very evident that CRA has much lesser Packet Forwarding Times per hop value compared to CAOR, LCOR and ExOR opportunistic protocols in Dynamic ad hoc networks with all three mobility models. This shows that CRA forwards a data packet minimum number of times for delivery over each hop in Dynamic ad hoc networks. From the results it is observed that the number of redundant data transmissions is very high for ExOR protocol. This is due to the link state style updating mechanism used by the protocol. Opportunistic protocols LCOR and CAOR also has value approximately between 1.5 and 2 which indicates duplicate data transmissions at intermediate nodes in the network.

From the graph it is evident that Forwarding Times Per Hop value of CRA is approximately equal to one that is every packet is forwarded only one time by each intermediate node in the network for delivery at the destination. It is also observed that CRA maintains this value even at higher speeds in the network. Thus it is verified from the results that CRA eliminates duplicate forwarding at intermediate nodes in the network and enhances the efficiency of opportunistic routing protocols in Dynamic ad hoc networks with extremely mobile nodes.

\subsubsection{Variation of Forwarding Times Per Hop with Network Dimension}

The Forwarding Times Per Hop value of the various protocols are measured with varying network dimensions. The speeds of the devices are varied in three network scenarios with the three mobility models respectively. 


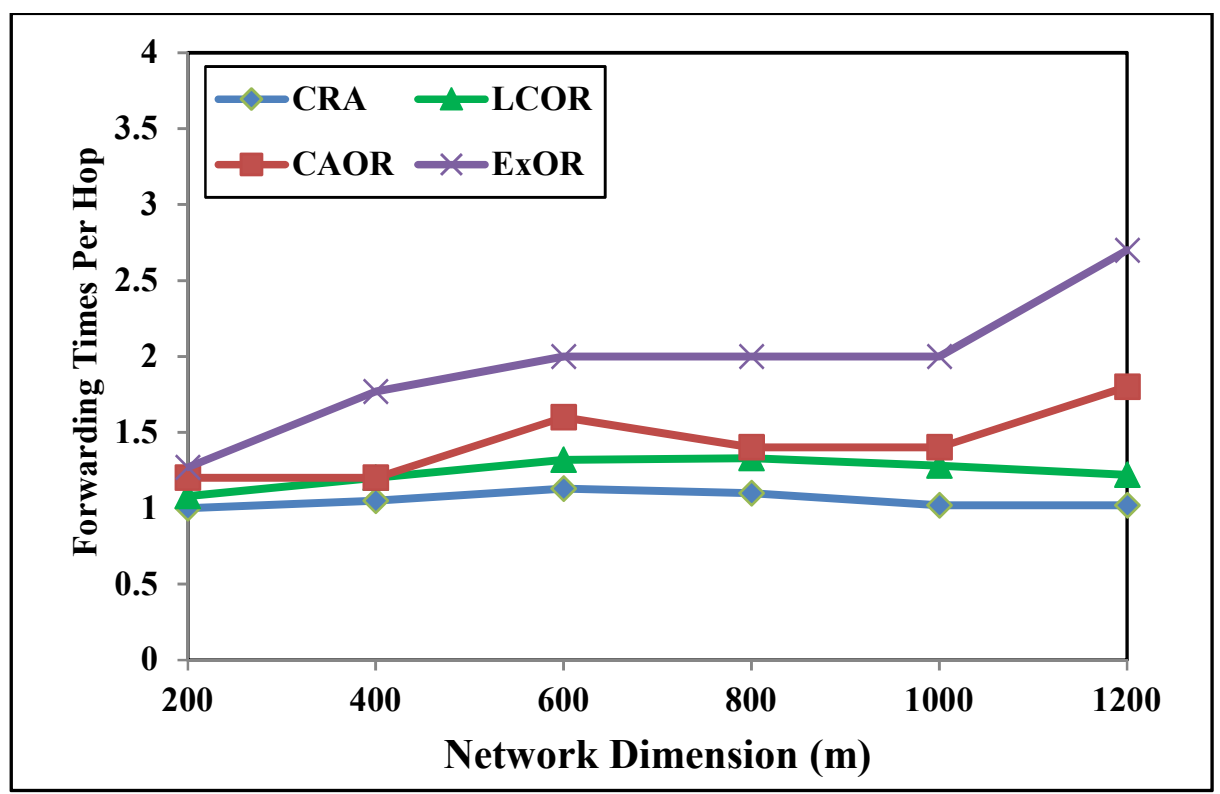

Figure 5 Forwarding Times Per Hop with Varying Network Dimension (Random Way Point Mobility Model)

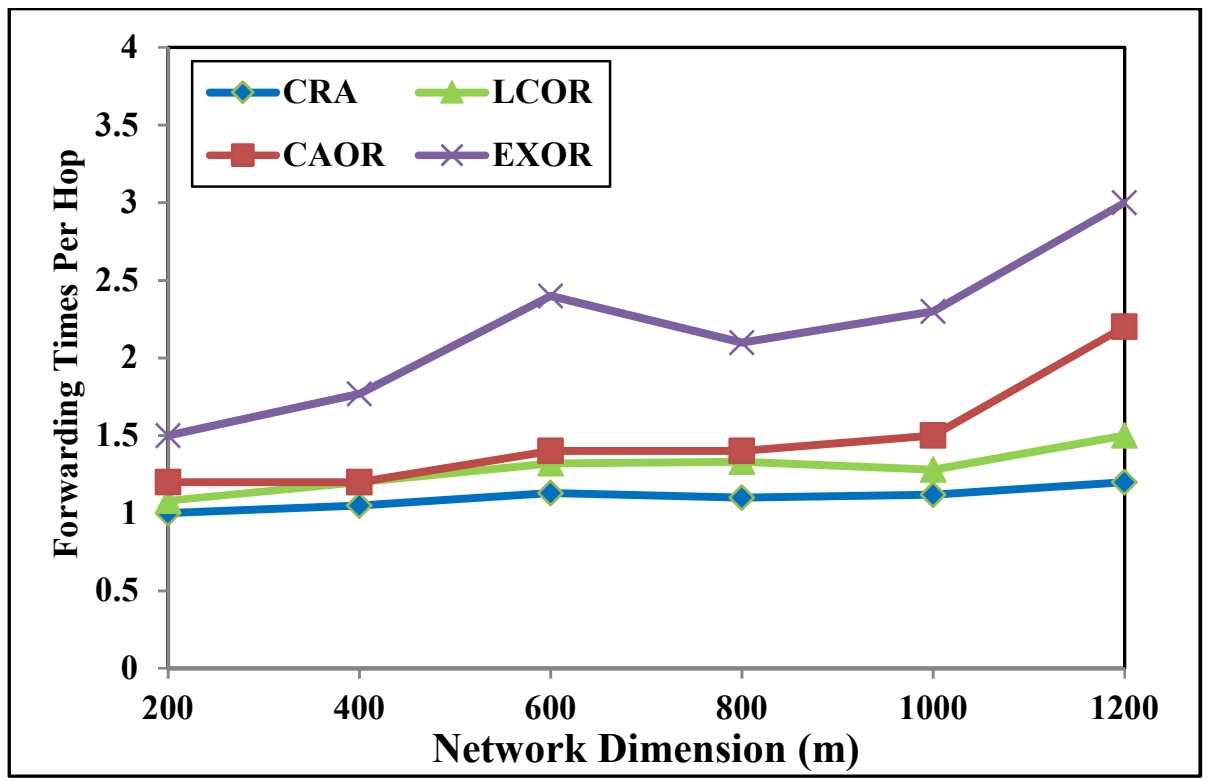

Figure 6 Forwarding Times Per Hop with Varying Network Dimension (Random Walk Mobility Model) 


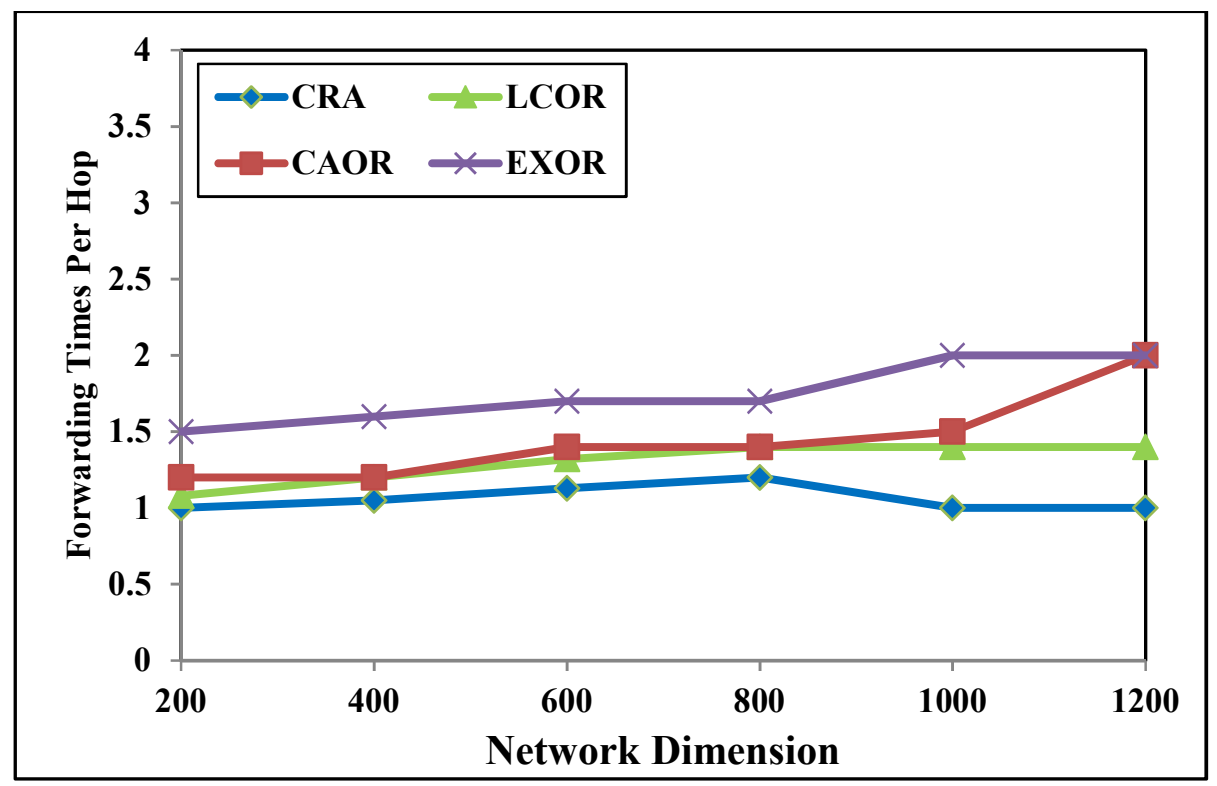

Figure 7 Forwarding Times Per Hop with Varying Network Dimension (Random Direction Mobility Model)

Figure 5, 6 and 7 shows the Forwarding Times Per Hop value with varying network size, obtained using Control Relaying with Acknowledgements, CAOR, LCOR and ExOR protocols in three simulations scenarios generated by random way point mobility model, random walk mobility model and random direction mobility model respectively. From the graph it is very evident that CRA has much lesser Forwarding Times Per Hop value compared to CAOR, LCOR and ExOR opportunistic protocols in Dynamic ad hoc networks with different network dimension and with all three mobility models. This validates that CRA forwards a data packet minimum number of times for delivery over each hop in scalable Dynamic ad hoc networks with large number of extremely dynamic nodes.

\subsubsection{Variation of Forwarding Times Per Hop with Packet Arrival Rate}

The Forwarding Times Per Hop value of the various protocols are measured with varying packet arrival rates from 10 packets per second to 50 packets per second. 


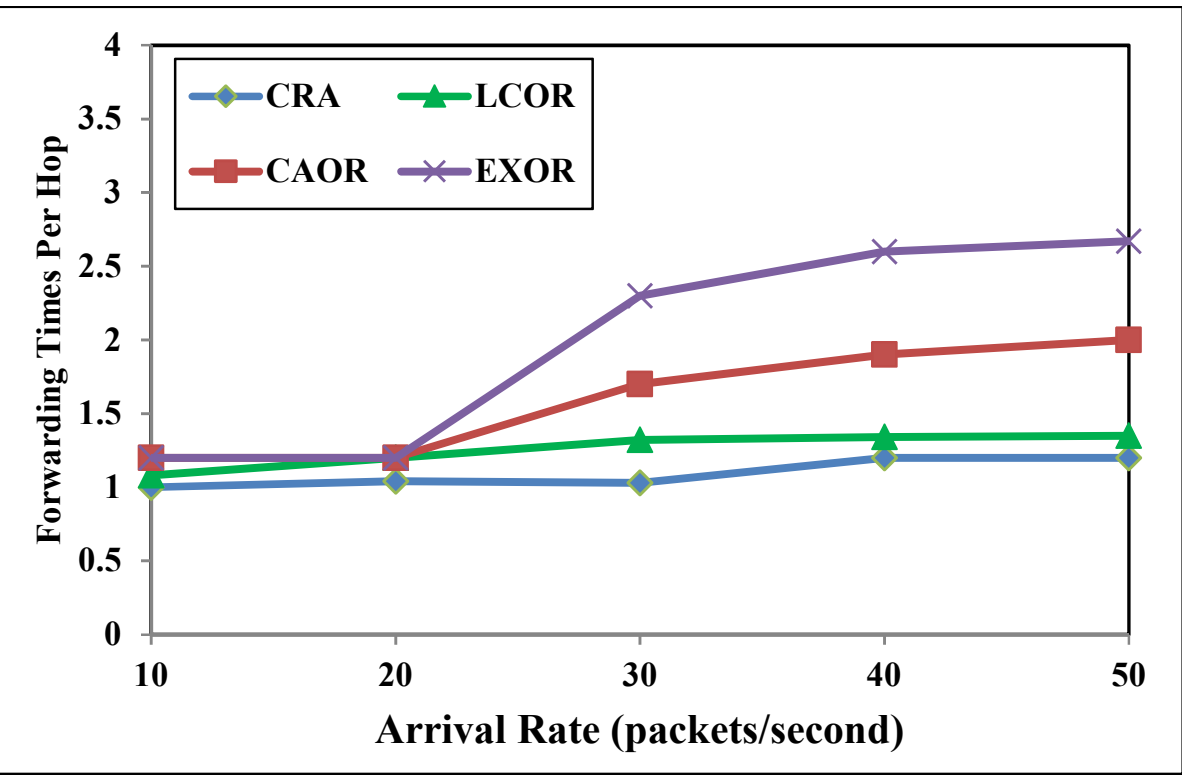

Figure 8 Forwarding Times Per Hop with Varying Arrival Rate (Random Way Point Mobility Model)

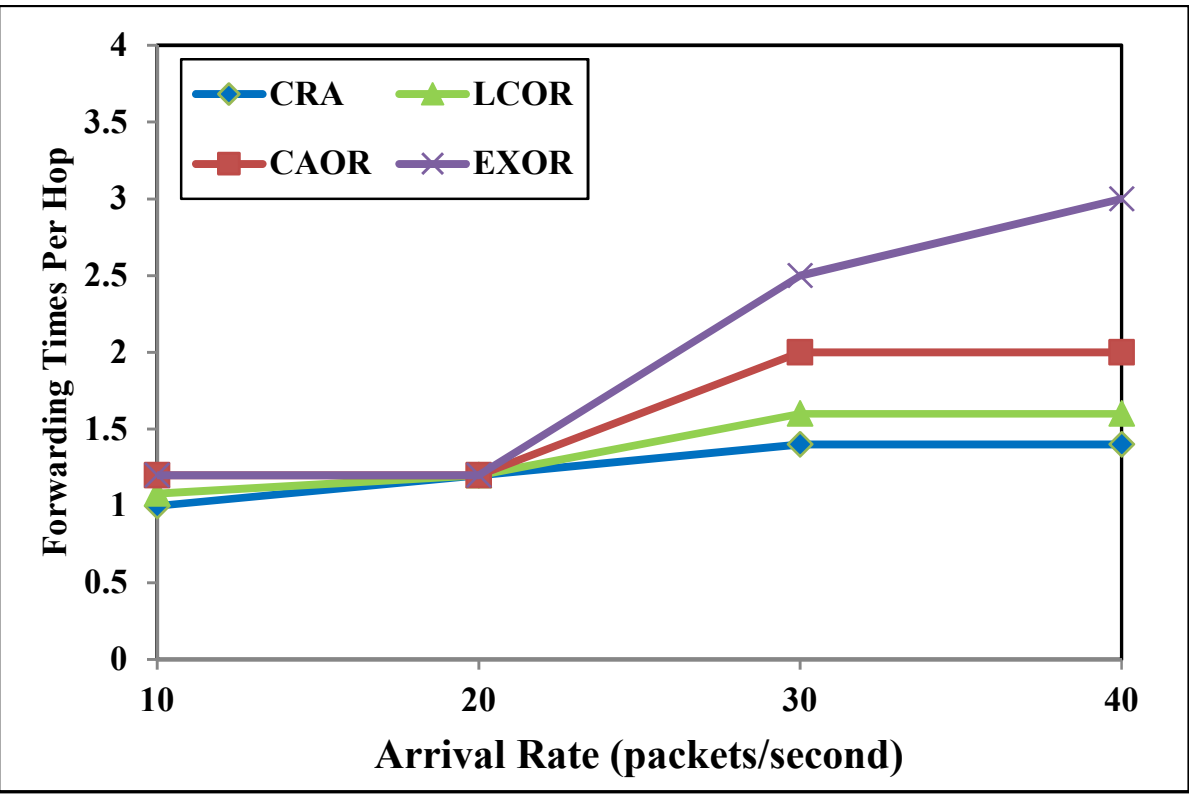

Figure 9 Forwarding Times Per Hop with Varying Arrival Rate (Random Walk Mobility Model) 


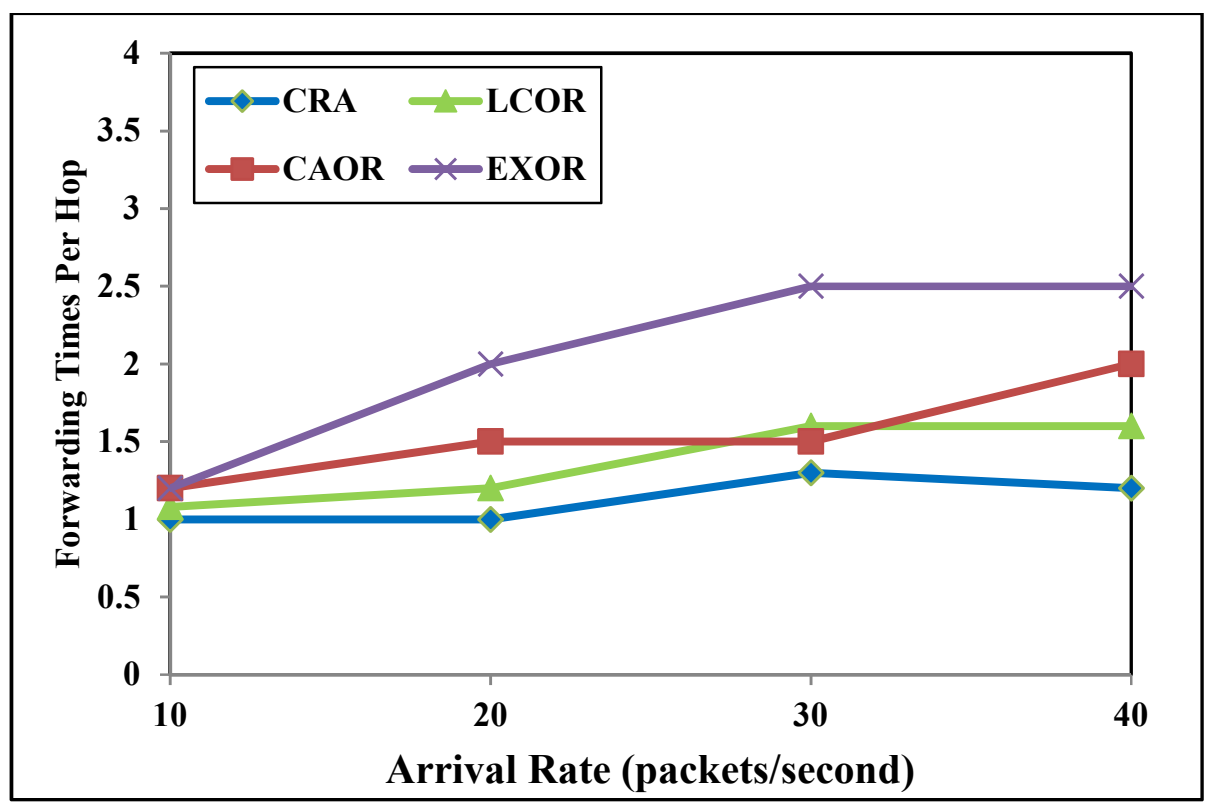

\section{Figure 10 Forwarding Times Per Hop with Varying Arrival rate (Random Direction Mobility Model)}

Figure 8, 9 and 10 shows the Forwarding Times Per Hop value with varying packet arrival rates in Dynamic ad hoc networks, achieved using Control Relaying with Acknowledgements, CAOR, LCOR and ExOR protocols in three simulations scenarios generated by random way point mobility model, random walk mobility model and random direction mobility model respectively.

From the graph it is very evident that CRA has much lesser Forwarding Times Per Hop value compared to CAOR, LCOR and ExOR opportunistic protocols in Dynamic ad hoc networks with different packet arrival rates and with all three mobility models. This proves the fact that CRA is able to provide efficient transmission in Dynamic ad hoc networks even with increasing arrival rates of data packets.

\subsubsection{Variation of Forwarding Times per Hop with Number of Nodes}

The performance of the proposed method is measured with varying number of nodes. 


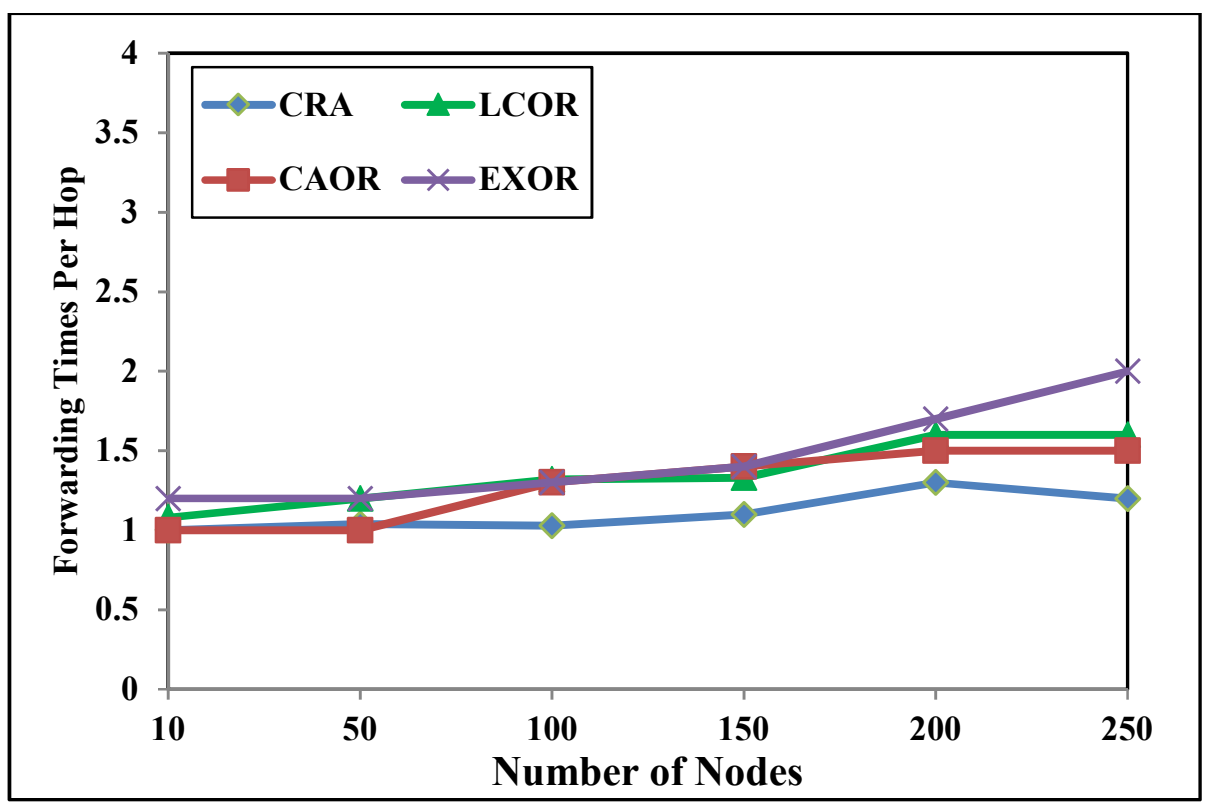

Figure 11 Forwarding Times Per Hop with Varying Number of Nodes (Random Way Point Mobility Model)

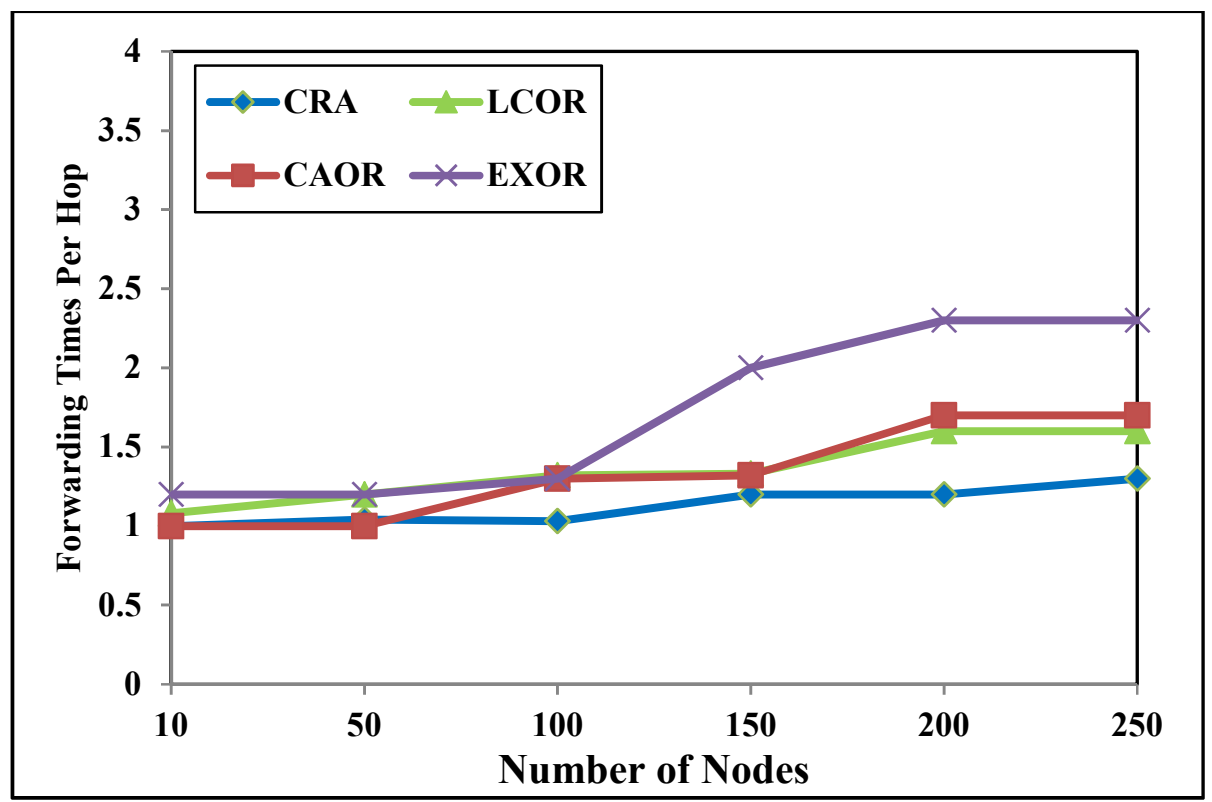

Figure 12 Forwarding Times Per Hop with Varying Number of Nodes (Random Walk Mobility Model) 


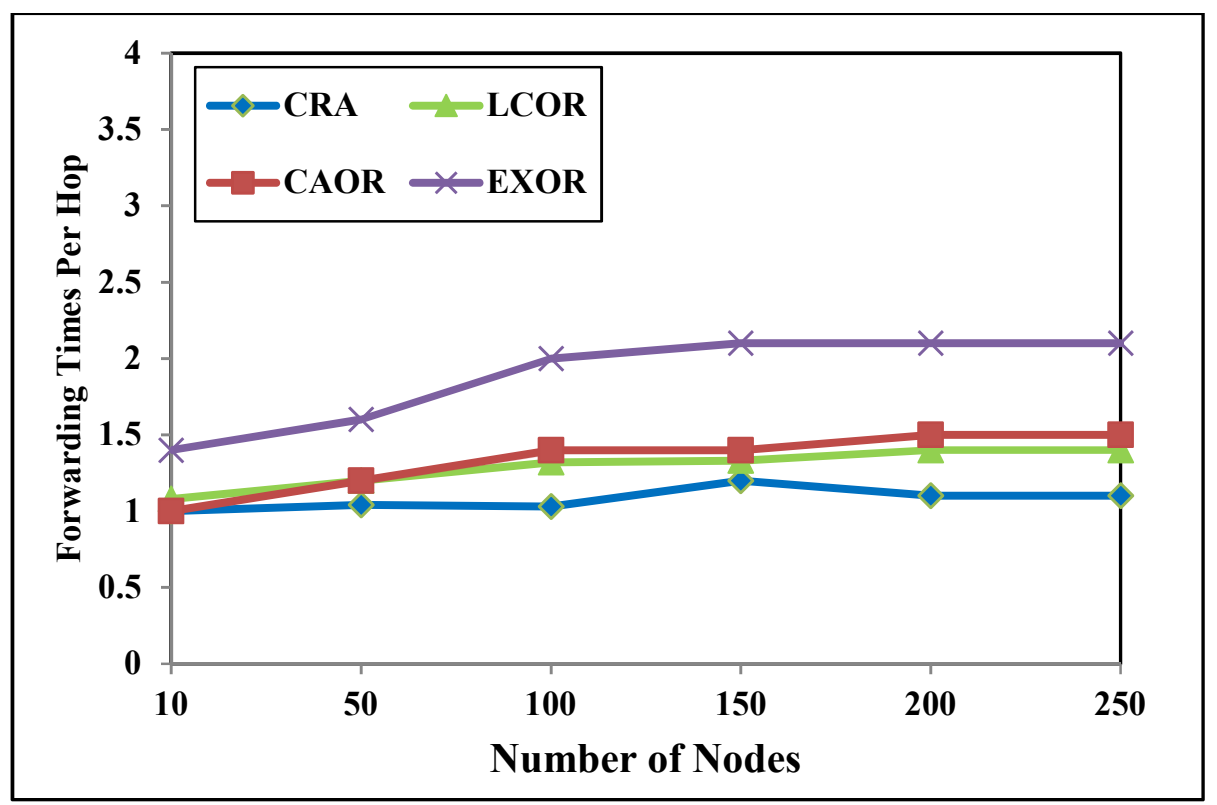

Figure 13 Forwarding Times Per Hop with Varying Number of Nodes (Random Direction Mobility Model)

Figure 11, 12 and 13 shows the Forwarding Times Per Hop valuewith varying number of nodes in Dynamic ad hoc networks, achieved using Control Relaying with Acknowledgements, CAOR, LCOR and ExOR protocols in three simulations scenarios generated by random way point mobility model, random walk mobility model and random direction mobility model respectively.

From the graph it is very evident that CRA has much lesser Forwarding Times Per Hop value compared to CAOR, LCOR and ExOR opportunistic protocols in Dynamic ad hoc networks with different number of nodes and with all three mobility models. This proves the fact that CRA is able to provide efficient transmission in Dynamic ad hoc networks even with increasing number of nodes in large networks. Thus results from simulations show that CRA maintains excellent Quality of Service in Dynamic ad hoc networks with varying speed of nodes, varying network dimensions, varying packet arrival rates and with increasing number of wireless devices. 


\section{CONCLUSION}

This research article discussed the working of the enhanced opportunistic forwarding mechanism, Control Relaying with Acknowledgements (CRA) in dynamic ad hoc networks with extremely mobile wireless devices. The proposed method Control Relaying with Acknowledgements (CRA) controlled the redundant relaying of data packets at each intermediate wireless device in the network. CRA worked per packet basis using information piggybacked on each packet. Only the devices that reply with an acknowledgement were considered for forwarding the data packet. Among the devices that reply with the acknowledgement, the device that was nearest to the destination was selected for forwarding. All the remaining devices do not act on the broadcasted data packet. Performance analysis of CRA was done with simulations in NS-2 with random way point, random walk and random direction mobility models. Forwarding Times Per Hop value was used for the performance comparison of Control Relaying with Acknowledgements (CRA) with other opportunistic protocols. This value measured the amount of duplicate transmission at intermediate nodes in the network. Results showed that CRA had a less Forwarding Times Per Hop value approximately equal to one confirming the elimination of redundant relaying of data packets at intermediate nodes by CRA. This also proved that every packet is forwarded only one time by each intermediate node in the network for delivery at the destination with CRA opportunistic forwarding method. The comparison of results confirmed that CRA eliminated redundant relaying of data packets at intermediate nodes and achieved excellent performance among all the major reliable opportunistic protocols in dynamic ad hoc networks with varying speed of nodes, varying network dimensions, varying packet arrival rates and varying number of nodes. In future we may also look at integrating this acknowledgement mechanism with Internet of Things and vehicular fog networks [35-40].

\section{References}

[1] S. Giordano and W. Lu, Challenges in Mobile Ad Hoc Networking, IEEE Communications Magazine, 39(6) (2001)129-129.

[2] I. Chlamtac, M. Conti and J. Liu, Mobile Ad Hoc Networking: Imperatives and Challenges, Ad Hoc Networks, 1(1) (2003) 13-64. 
[3] E. Gelenbe, Quality of Service in Ad Hoc Networks, Ad Hoc Networks, 2(3) (2004) 203204.

[4] J. Hoebeke, I. Moerman, B. Dhoedt and P. Demeester, An Overview of Mobile Ad Hoc Networks: Applications and Challenges, Journal of The Communications Network, 3(3) (2004) pp.60-66.

[5] M. Conti, and S. Giordano, Mobile Ad Hoc Networking: Milestones, Challenges, and New Research Directions, IEEE Communications Magazine, 52(1) (2014) 85-96.

[6] V. G. Menon and P M J Prathap, Performance of various Routing Protocols in Mobile A Hoc Networks-A Survey, Research Journal of Applied Sciences, Engineering and Technology, 6(22), pp. 4181-4185, December 2013.

[7] V. G. Menon and P M J Prathap, "Routing in Highly Dynamic Ad Hoc Networks: Issues and Challenges", International Journal of Computer Science and Engineering, vol.8, no. 4, pp.112-116, 2016.

[8] V. G. Menon, Sreekala C S, Vibin J, Teenu T., Eldho A, "Performance Analysis of Traditional Topology based Routing Protocols in Mobile Ad hoc Networks"The International Journal of Computer Science Applications,vol. 2,no.1, pp. 1-6.

[9] V. G. Menon and P M J Prathap. (2017). Towards Optimal Data Delivery in Highly Mobile Wireless Ad Hoc Networks. International Journal of Computer Science and Engineering, 9(1), 1-6.

[10] V. G. Menon, "Analyzing the Performance of Random Mobility Models with Opportunistic Routing." Volume 10, Number 5 (2017), pp. 1221-1226.

[11] Biswas, S. and Morris, R. (2005), "ExOR: Opportunistic Multi-Hop Routing for Wireless Networks", Proceedings of the 2005 Conference on Applications, Technologies, Architectures, and Protocols for Computer Communications (SIGCOMM '05), ACM, New York, USA, pp. 133-144.

[12] Bruno, R., Conti, M. and Nurchis, M. (2010), "Opportunistic Packet Scheduling and Routing in Wireless Mesh Networks", Proceedings of IFIP Wireless Days Conference, Venice, Italy, pp. 1-6.

[13] Perkins, C. E. and Bhagwat, P. (1994), "Highly Dynamic Destination-Sequenced Distance-Vector Routing (DSDV) for Mobile Computers", Proceedings of the Conference on Communications Architectures, Protocols and Applications (SIGCOMM '94). ACM, New York, USA, pp. 234-244.

[14] Jacquet, P., Muhlethaler, P. and Qayyum, A. (1998), "Optimized Link State Routing Protocol", Internet Draft, Available at draft-ietf-manetolsr-00.txt. 
[15] Bellur, B., Ogier, R.G. and Templin, F.L. (2001), "Topology Broadcast Based on Reverse-Path Forwarding (TBRPF)", IETF Internet Draft, Available at draft-ietf-manettbrpf-01.txt.

[16] Johnson, D. B. and Maltz, D. A. (1996), "Dynamic Source Routing in Ad Hoc Wireless Networks", Mobile Computing, Kluwer Academic Publishers, vol. 353, Chapter 5, pp. 153-181.

[17] Karp, B. and Kung, H. T. (2000), “GPSR: Greedy Perimeter Stateless Routing for Wireless Networks", Proceedings of The 6th Annual International Conference on Mobile Computing and Networking (MobiCom '00), ACM, New York, USA, pp. 243-254.

[18] Mauve, M., Widmer, J., and Hartenstein, H. (2001), "A Survey on Position-Based Routing in Mobile Ad Hoc Networks”, IEEE Network, vol. 15, no.6, pp. 30-39.

[19] Cadger, F., Curran, K., Santos J and Moffett, S (2013), “A Survey of Geographical Routing in Wireless Ad-Hoc Networks", IEEE Communications Surveys and Tutorials, vol. 15, no. 2, pp. 621-653.

[20] V. G. Menon, Jogi Priya P M, Joe Prathap P M, "Analyzing the behavior and performance of greedy perimeter stateless routing protocol in highly dynamic mobile ad hoc networks", Life Science Journal, 10(2): pp 1601-1605, 2013.

[21] V. G. Menon and Joe Prathap P.M, "Performance analysis of geographic routing protocols in highly mobile ad hoc network," Journal of Theoretical and Applied Information Technology, vol. 54 no. 1, pp 127-133, 2013.

[22] Chakchouk, N. (2015), "A Survey on Opportunistic Routing in Wireless Communication Networks", IEEE Communications Surveys and Tutorials, vol. 17, no. 4, pp. 2214-2241.

[23] Menon V. G. and Joe Prathap P M, (2016), "Comparative Analysis of Opportunistic Routing Protocols for Underwater Acoustic Sensor Networks" Proceedings of the IEEE International Conference on Emerging Technological Trends, Kerala, India.

[24] Menon V G, "Opportunistic Routing Protocols in Underwater Acoustic Sensor Networks: Issues, Challenges, and Future Directions", Magnetic Communications: From Theory to Practice, CRC Press, pp. 127-148, 2018

[25] Menon V G and Joe Prathap PM, "Moving From Topology-Dependent to Opportunistic Routing Protocols in Dynamic Wireless Ad Hoc Networks: Challenges and Future Directions", Algorithms, Methods, and Applications in Mobile Computing and Communications, IGI Global, 1-23

[26] Menon V G and Joe Prathap P M, "A Review on Efficient Opportunistic Forwarding Techniques used to Handle Communication Voids in Underwater Wireless Sensor Networks, Advances in Wireless and Mobile Communications, vol 10, no. 5, pp. 10591066,2017 
[27] V. G. Menon and Joe Prathap P.M, "Analysing the Behaviour and Performance of Opportunistic Routing Protocols in Highly Mobile Wireless Ad Hoc Networks", International Journal of Engineering and Technology, vol. 8, no. 5, pp. 19161924, 2016.

[28] V. G. Menon, Joe Prathap P M and Vijay A, "Eliminating Redundant Relaying of Data Packets for Efficient Opportunistic Routing in Dynamic Wireless Ad Hoc Networks", Asian Journal of Information Technology, vol. 12, no.17, 2016

[29] V. G. Menon, "Survey on Latest Energy Based Routing Protocols for Underwater Wireless Sensor Networks", International Journal of Computer Networks and Wireless Communications (IJCNWC), Vol.6, No 6, Nov-Dec 2016, pp-52-55.

[30] V. G. Menon and P. M. Joe Prathap, "Opportunistic routing with virtual coordinates to handle communication voids in mobile ad hoc networks," in Advances in Signal Processing and Intelligent Recognition Systems, vol. 425 of Advances in Intelligent Systems and Computing, pp. 323-334, Springer International, 2016.

[31] V. G. Menon, Joe Prathap Pathrose, and Jogi Priya, "Ensuring Reliable Communication in Disaster Recovery Operations with Reliable Routing Technique," Mobile Information Systems, vol. 2016, Article ID 9141329, 10 pages, 2016.

[32] Menon, V. Optimized Opportunistic Routing in Highly Dynamic Ad hoc Networks. Preprints 2019, 2019020130

[33] Zhao, Z., Rosario, D., Braun, T., Cerqueira, E., (2014), "Context-Aware Opportunistic Routing in Mobile Ad-Hoc Networks Incorporating Device Mobility", Proceedings of the IEEE Wireless Communications and Networking Conference, Istambul, Turkey, pp. $2138-2143$.

[34] Dubois-Ferriere, H., Grossglauser, M. and Vetterli, M. (2007), "Least-Cost Opportunistic Routing", Proceedings of 2007 Allerton Conference on Communication, Control, and Computing, Allerton, UK, pp. 1-8.

[35] Menon, Varun G., and Joe Prathap. "Vehicular Fog Computing: Challenges Applications and Future Directions." International Journal of Vehicular Telematics and Infotainment Systems (IJVTIS) 1.2 (2017): 15-23.

[36] Menon V G, Moving From Vehicular Cloud Computing to Vehicular Fog Computing: Issues and Challenges International Journal on Computer Science and Engineering, vol. 9, no. 2, pp. 14-18, 2017.

[37] Deshkar, S., Thanseeh, R. A., \& Menon, V. G. (2017). A Review on IoT based m-Health Systems for Diabetes. International Journal of Computer Science and Telecommunications, 8(1), 13-18. 
[38] Philip, V., Suman, V. K., Menon, V. G., \& Dhanya, K. A. (2017). A Review on latest Internet of Things based Healthcare Applications. International Journal of Computer Science and Information Security, 15(1), 248.

[39] Vinoj, P. G., Jacob, S., \& Menon, V. G. (2018, September). Hybrid brainactuated muscle interface for the physically disabled. In BASIC \& CLINICAL PHARMACOLOGY \& TOXICOLOGY (Vol. 123, pp. 8-9). 111 RIVER ST, HOBOKEN 07030-5774, NJ USA: WILEY.

[40] G. Chen, J. Tang and J. P. Coon, "Optimal Routing for Multihop Social-Based D2D Communications in the Internet of Things," in IEEE Internet of Things Journal, vol. 5, no. 3, pp. 1880-1889, June 2018 